\title{
A FUNDAMENTAL INVARIANT OF THE DIS- CONTINUOUS $\zeta$-GROUPS DEFINED BY THE NORMAL CURVES OF ORDER $n$ IN A SPACE OF $n$ DIMENSIONS.
}

BY PROFESSOR J. W. YOUNG.

(Read before the American Mathematical Society, February 29, 1908.)

A NORMAL curve $C_{n}$ of order $n$ in a space $S_{n}$ of $n$ dimensions is transformed into itself by $\infty^{3}$ collineations

$$
z_{i}^{\prime}=\sum_{k=0}^{n} a_{i k} z_{k} \quad(i=0,1, \cdots, n)
$$

in $S_{n}$. Each of these collineations subjects the parameter $\zeta_{1}: \zeta_{2}$ of $C_{n}^{n}$ to a linear substitution $\zeta_{1}^{\prime}=\alpha \zeta_{1}+\beta \zeta_{2}, \zeta_{2}^{\prime}=\gamma \zeta_{1}+\delta \zeta_{2}$. The group of collineations obtained by restricting the coefficients $a_{i k}$ to rational integral values with determinant $\left|a_{i k}\right|=1$ is properly discontinuous, and the corresponding group $\Gamma_{n}$ of linear fractional substitutions

$$
\zeta^{\prime}=\frac{\alpha \zeta+\beta}{\gamma \zeta+\delta} \quad(\alpha \delta-\beta \gamma=1)
$$

on the parameter $\zeta=\zeta_{1}: \zeta_{2}$ is hence likewise properly discontinuous. This latter group $\Gamma_{n}$ we call the discontinuous $\zeta$-group defined by $C_{n}$.

The arithmetic definition of discontinuous groups of substitutions (1) forms one of the fundamental problems in the theory of automorphic functions. The above is an outline of one of the few effective methods that have been suggested for this purpose ; $*$ but on account of its complexity little has been done with it for values of $n>2$. $\dagger$ General results applying to any value of $n$ are almost totally lacking. The case $n=2$. however, has been exhaustively treated on the arithmetic side by Fricke, $\downarrow$ and has yielded results of great importance. Funda-

* Fricke, Chicago Congress Papers, p. 85, and J. W. Young, "On a class of discontinuous $\zeta$-groups, etc.," Rendiconti del Circolo matematico di Palermo, vol. $23(1907)$, p. 97 .

$\dagger$ The case $n=4$ has been partially treated by the author, loc. cit.

Fricke, "Ueber indefinite Formen mit drei und vier Variabeln," Göttinger Nachrichten, Dec. 13, 1883. Cf. also Fricke-Klein, "Theorie der automorphen Functionen" (Leipzig, 1897), vol. 1, p. $502 \mathrm{ff}$. 
mental in Fricke's treatment of the groups $\Gamma_{2}$ is an invariant $J=(\alpha+\delta)^{2}-1$, from the consideration of which follows that any group $\Gamma_{2}$ contains no elliptic substitutions of periods other than $2,3,4$, and $6 . *$ It is the object of this paper to calculate the corresponding invariant for the groups $\Gamma_{n}$ in general, and to derive the corresponding general theorem concerning the existence of elliptic substitutions in any $\Gamma_{n}$.

The equations of any normal curve $C_{n}$ can by linear transformation be reduced to the form $z_{i}=\zeta_{1}^{n-i} \zeta_{2}^{i}(i=0,1, \ldots, n)$. We proceed first to derive the most general collineation in $S_{n}$ which leaves this curve invariant. It is readily obtained by subjecting the parameter $\zeta_{1}: \zeta_{2}$ of the curve to the substitution

This gives

$$
\zeta_{1}^{\prime}=\alpha \zeta_{1}+\beta \zeta_{2}, \quad \zeta_{2}^{\prime}=\gamma \zeta_{1}+\delta \zeta_{2} \quad(\alpha \delta-\beta \gamma=1) .
$$

$$
z_{i}^{\prime}=\left(\alpha \zeta_{1}+\beta \zeta_{2}\right)^{n-i}\left(\gamma \zeta_{1}+\delta \zeta_{2}\right)^{i} \quad(i=0,1, \ldots, n) .
$$

If the right-hand member be expanded and the products $\zeta_{1}^{\lambda} \xi_{2}^{\mu}$ replaced by their equivalent $z_{j}^{\text {'s, }}$, the desired collineation is obtained in the form

(2) $z_{i}^{\prime}=\sum_{k=0}^{n} a_{i k} z_{k}=\sum_{j=0}^{n-i} \sum_{j^{\prime}=0}^{i}\left(\begin{array}{c}n-i \\ j\end{array}\right)\left(\begin{array}{c}i \\ j^{\prime}\end{array}\right) \alpha^{n-i-j} \beta^{j} \gamma^{i-j^{\prime}} \delta^{j^{\prime}} z_{j+j^{\prime}}$.

The collineation which transforms any $C_{n}$ whatever into itself is then obtained from this by linear transformation, since all $C_{n}^{\text {'s }}$ are projectively equivalent. However, it is well-known that under every such transformation the sum $\Sigma a_{i i}$ of the leading diagonal elements of the matrix $\left(a_{i k}\right)$ is an absolute invariant. This invariant, which we will denote by $J$, turns out to be a polynomial in $\alpha+\delta$ with integral coefficients. It may be calculated as follows :

From (2) we have at once, since $a_{i i}$ is the coefficient of $z_{i}$,

$$
J=\sum_{i=0}^{n} a_{i i}=\sum_{i=0}^{n} \sum_{j=0}^{n-i}\left(\begin{array}{c}
n-i \\
j
\end{array}\right)\left(\begin{array}{c}
i \\
i-j
\end{array}\right) \alpha^{n-i-j} \delta^{i-j} \beta^{j} \gamma^{j} .
$$

If in this expression we replace $\beta \gamma$ by $\alpha \delta-1$, we obtain

$$
\begin{array}{r}
J=\sum_{i=0}^{n} \sum_{j=0}^{n-i}\left(\begin{array}{c}
n-i \\
j
\end{array}\right)\left(\begin{array}{c}
i \\
i-j
\end{array}\right) \alpha^{n-i-j} \delta^{i-j} \sum_{\nu=0}^{j}(-1)^{\nu}\left(\begin{array}{l}
j \\
\nu
\end{array}\right) \alpha^{j-\nu} \delta^{j-\nu} \\
=\sum_{i=0}^{n} \sum_{j=0}^{n-i} \sum_{\nu=0}^{j}(-1)^{\nu}\left(\begin{array}{c}
n-i \\
j
\end{array}\right)\left(\begin{array}{c}
i \\
i-j
\end{array}\right)\left(\begin{array}{l}
j \\
\nu
\end{array}\right) \alpha^{n-i-\nu} \delta^{i-\nu} .
\end{array}
$$

* Fricke-Klein, loc. cit., p. 517. 
Now we readily verify the following relations between binomial coefficients :

$$
\left(\begin{array}{l}
p \\
j
\end{array}\right)\left(\begin{array}{l}
j \\
q
\end{array}\right)=\left(\begin{array}{l}
p \\
q
\end{array}\right)\left(\begin{array}{c}
p-q \\
j-q
\end{array}\right)=\left(\begin{array}{l}
p \\
q
\end{array}\right)\left(\begin{array}{l}
p-q \\
p-j
\end{array}\right) .
$$

The first of these gives

$$
\left(\begin{array}{c}
n-i \\
j
\end{array}\right)\left(\begin{array}{l}
j \\
\nu
\end{array}\right)=\left(\begin{array}{c}
n-i \\
\nu
\end{array}\right)\left(\begin{array}{c}
n-i-\nu \\
j-\nu
\end{array}\right)
$$

whence substituting we have

$$
J=\sum_{i} \sum_{j} \sum_{\nu}(-1)^{\nu}\left(\begin{array}{c}
n-i \\
\nu
\end{array}\right)\left(\begin{array}{c}
i \\
i-j
\end{array}\right)\left(\begin{array}{c}
n-i-\nu \\
j-\nu
\end{array}\right) \alpha^{n-i-\nu} \delta^{i-\nu} .
$$

Here and in all that follows, the summations are to be extended over all possible combinations of the symbols involved. The order in which the summations are carried out is clearly immaterial. In the last expression for $J$ we replace $\left(\begin{array}{c}i \\ i-j\end{array}\right)$ by $\left(\begin{array}{l}i \\ j\end{array}\right)$ and $j$ by $\nu+j^{\prime}$ and sum first with respect to $j^{\prime}$, making use of the formula*

We thus obtain

$$
\sum_{s=0}\left(\begin{array}{c}
p \\
n+s
\end{array}\right)\left(\begin{array}{l}
q \\
s
\end{array}\right)=\left(\begin{array}{c}
p+q \\
n+q
\end{array}\right)
$$

$$
\begin{aligned}
J=\sum_{i} \sum_{\nu} \sum_{j^{\prime}}(-1)^{\nu}\left(\begin{array}{c}
n-i \\
\nu
\end{array}\right)\left(\begin{array}{c}
i \\
\nu+j^{\prime}
\end{array}\right)\left(\begin{array}{c}
n-i-\nu \\
j^{\prime}
\end{array}\right) \alpha^{n-i-\nu} \delta^{i-\nu} \\
=\sum_{i} \sum_{\nu}(-1)^{\nu}\left(\begin{array}{c}
n-i \\
\nu
\end{array}\right)\left(\begin{array}{l}
n-\nu \\
n-i
\end{array}\right) \alpha^{n-i-\nu} \delta^{i-\nu} .
\end{aligned}
$$

By the second part of (3), this may be written

$$
J=\sum_{\nu} \sum_{i}(-1)^{\nu}\left(\begin{array}{c}
n-\nu \\
\nu
\end{array}\right)\left(\begin{array}{c}
n-2 \nu \\
i-\nu
\end{array}\right) \alpha^{n-i-\nu} \delta^{i-\nu},
$$

which is clearly equivalent to

$$
J=\sum_{\nu=0}(-1)^{\nu}\left(\begin{array}{c}
n-\nu \\
\nu
\end{array}\right)(\alpha+\delta)^{n-2 \nu} .
$$

This is the desired invariant. For the value $n=2$ it evidently reduces to the expression already referred to for this case. The result obtained may be stated as follows:

* Netto, Lehrbuch der Combinatorik, Leipzig, 1901, p. 250. 
Theorem 1. If the parameter $\zeta$ of any normal curve of order $n$ in a space $S_{n}$ of $n$ dimensions is transformed by a substitution (1), the sum $J$ of the elements in the leading diagonal of the matrix of the corresponding collineation in $S_{n}$ is independent of the curve and is given by (4).

This yields at once a condition on the periods of the elliptic substitutions in any discontinuous group $\Gamma_{n}$ obtained by restricting the coefficients in the collineations to rational integral values. For every substitution (1) of any such group $\Gamma_{n}$, the number $J$ must be an integer. Now, if $\omega$ be the period of any elliptic substitution (1) in a $\Gamma_{n}$ we have*

$$
\pm(\alpha+\delta)=2 \cos \frac{\pi}{\omega} \text {. }
$$

If this expression be substituted in (4), remembering that $J$ is an integer, we obtain an equation which $\omega$ must satisfy. This may be put into a more convenient form by placing $x=e^{\pi i / \omega}$, and hence placing $(\alpha+\delta)=x+x^{-1} \cdot \dagger$ We obtain readily

$$
J=\sum_{\nu} \sum_{\mu}(-1)^{\nu}\left(\begin{array}{c}
n-\nu \\
\nu
\end{array}\right)\left(\begin{array}{c}
n-2 \nu \\
\mu
\end{array}\right) x^{n-2 \nu-2 \mu},
$$

or placing $\nu+\mu=p$, we obtain the coefficient of $x^{n-2 p}$ in the form

$$
\sum_{\nu}(-1)^{\nu}\left(\begin{array}{c}
n-\nu \\
\nu
\end{array}\right)\left(\begin{array}{c}
n-2 \nu \\
p-\nu
\end{array}\right)
$$

By applying the first relation (3) in the opposite direction, we obtain

$$
\left(\begin{array}{c}
n-\nu \\
\nu
\end{array}\right)\left(\begin{array}{c}
n-2 \nu \\
p-\nu
\end{array}\right)=\left(\begin{array}{c}
n-\nu \\
p
\end{array}\right)\left(\begin{array}{l}
p \\
\nu
\end{array}\right)
$$

But by a well-known relation we have $\$$

$$
\sum_{\nu}(-1)^{\nu}\left(\begin{array}{c}
n-\nu \\
p
\end{array}\right)\left(\begin{array}{l}
p \\
\nu
\end{array}\right)=\left(\begin{array}{l}
n-p \\
n-p
\end{array}\right)=1
$$

We have therefore the relation

$$
J=x^{n}+x^{n-2}+x^{n-4}+\cdots+x^{-n+2}+x^{-n},
$$

* Fricke-Klein, loc. cit., p. 517.

$\dagger$ The signs of $a, \delta$ can always be so chosen that the upper sign in (5) holds.

$\ddagger$ Netto, loc. cit., p. 252. 
or multiplying by $x^{n}$,

$$
x^{2 n}+x^{2 n-2}+x^{2 n-4}+\cdots+x^{2}+1=J x^{n} .
$$

Since $x^{2} \neq 1$, this may also be written

$$
x^{2 n+2}-1=J x^{n}\left(x^{2}-1\right) .
$$

A still more convenient form of this equation, for some purposes, may be obtained by placing $x=e^{\pi i / \omega}=\cos \omega+i \sin \omega$ and equating real and imaginary parts. By means of elementary transformations (6) is then readily shown to be equivalent to the equation

$$
\sin \frac{(n+1) \pi}{\omega}=J \sin \frac{\pi}{\omega} .
$$

These results may be stated as follows:

Theorem 2. In any discontinuous $\zeta$-group defined by a normal curve $C_{n}$ in a space $S_{n}$ the period $\omega$ of any elliptic substitution must satisfy an equation (7), where $J$ is an integer, or (6), where $x=e^{\pi i / \omega}$ and $J$ is an integer.

The numbers 2, 3 are possible periods for every value of $n$, as may be easily verified from the above condition. This follows also at once from the fact that the elliptic modular group, which contains substitutions of periods 2 and 3 , is a $\Gamma_{n}$ for every value of $n$. In fact it is the group defined by the canonical $C_{n}$ which we used above to obtain the desired invariant. \& It has already been pointed out that the only other possible periods in the case $n=2$ are 4 and 6 . As examples of the application of the last theorem we give in the table below the possible periods for the values of $n$ from 3 to 6 inclusive, calculated from (6):

Possible periods.

$$
\begin{array}{ll}
n=3 & 2,3,4,5 ; \\
n=4 & 2,3,4,5,6 ; \\
n=5 & 2,3,5,6,7 ; \\
n=6 & 2,3,4,6,7,8 .
\end{array}
$$

Moreover, by placing $J=0$ and \pm 1 , it is readily seen that every factor of $n, n+1$, and $n+2$ is a possible period in any $\Gamma_{n}$.

PRinceton University, February, 1908.

\& Cf. Young, loc. cit., p. 99, footnote. 